\title{
Propuesta de una planta para el tratamiento de residuos orgánicos en la ciudad de Tambogrande
}

\author{
Design of a plant for the treatment of organic waste in the city of \\ Tambogrande
}

\section{Proposta de estação de tratamento de resíduos orgânicos no município de Tambogrande}

\author{
Jorge Flores \\ jorgeflores20@gmail.com \\ ORCID 0000-0002-0633-7101 \\ Universidad Alas Peruanas, Perú
}

Artículo recibido enero 2021 | Arbitrado en febrero 2021 | Publicado en mayo 2021

RESUMEN

Uno de los problemas en las ciudades es el manejo de residuos orgánicos, puesto que se generan grandes volúmenes de estos y no hay una separación de los mismos en los puntos de generación. De manera que, el inicio de una solución a este problema, es separar la basura en residuos inorgánicos, residuos orgánicos y residuos sanitarios, para después ser recolectados y darles un tratamiento posterior, buscando el desarrollo sustentable en la comunidad. Es así, como el presente artículo tiene como objetivo proponer la instalación de una planta de tratamiento de residuos sólidos en la ciudad de Tambogrande. El tipo de investigación aplicada fue descriptiva, dado que se presentan algunas características fundamentales del fenómeno y se consideró adicionalmente cuasi experimental. El diseño de la planta está basado en tres ejes: ambiental, económico y social. Se obtiene un modelo de aprovechamiento de residuos sólidos orgánicos, mediante las técnicas de tratamiento biológico de compostaje.

Palabras clave: Compostaje; planta de tratamiento; residuos orgánicos; Tambogrande; técnicas de tratamiento

ABSTRACT

RESUMO

One of the problems in cities is the management of organic waste, since large volumes of these are generated and there is no separation of them at the generation points. So, the beginning of a solution to this problem is to separate the garbage into inorganic waste, organic waste and sanitary waste, to later be collected and given a subsequent treatment, seeking sustainable development in the community. Thus, the objective of this article is to propose the installation of a solid waste treatment plant in the city of Tambogrande. The type of applied research was descriptive, since some fundamental characteristics of the phenomenon are presented and it was additionally considered quasiexperimental. The design of the plant is based on three environmental, economic and social axes. A model of use of solid organic waste is obtained, in the techniques of biological composting treatment.

Key words: Composting; treatment plant, organic waste, Tambogrande, treatment techniques
Um dos problemas das cidades é o gerenciamento dos resíduos orgânicos, uma vez que grandes volumes deles são gerados e não há separação nos pontos de geração. Assim, o início de uma solução para esse problema é separar o lixo em resíduo inorgânico, resíduo orgânico e resíduo sanitário, para posteriormente ser coletado e tratado posteriormente, visando o desenvolvimento sustentável da comunidade. É assim que este artigo tem como objetivo propor a instalação de uma estação de tratamento de resíduos sólidos no município de Tambogrande. 0 tipo de pesquisa aplicada foi descritivo, visto que são apresentadas algumas características fundamentais do fenômeno e, adicionalmente, foi considerada quase experimental. 0 projeto da planta é baseado em três eixos: ambiental, econômico e social. Um modelo de aproveitamento de resíduos sólidos orgânicos é obtido por meio de técnicas de tratamento de compostagem biológica.

Palavras-chave: Compostagem, estação de tratamento, resíduos orgânicos, Tambogrande, técnicas de tratamento 
INTRODUCCIÓN

La contaminación ambiental es uno de los principales problemas que enfrenta el mundo, generando daños económicos, sociales y ambientales. Pero existen formas de tratar los residuos sólidos, como es el compostaje, que permiten el aprovechamiento de los residuos orgánicos, mejorando la calidad de las tierras y disminuyendo la contaminación.

Según Barradas (2009), durante el compostaje, el residuo se somete a una degradación bioxidativa bajo condiciones controladas, y como producto final se obtiene el compost. Los métodos de compostaje se pueden clasificar en sistemas abiertos y sistemas cerrados. En los sistemas abiertos se encuentran el composteo en "pilas" y el vermicompostaje (composteo con lombrices). En los sistemas cerrados se encuentran los reactores, que pueden ser aerobios (con presencia de oxígeno) o anaerobios (ausencia de oxígeno). Los reactores aerobios son utilizados para producir composta como producto, mientras que los reactores anaerobios (generalmente llamados biodigestores) tienen como producto principal, el gas metano.

En estos sistemas, la fase inicial de fermentación se realiza en reactores que pueden ser de dos tipos: horizontales o verticales, mientras que la fase final de maduración se hace al aire libre o en naves abiertas. Son sistemas desarrollados para reducir considerablemente las superficies de compostaje y lograr un mejor control de los parámetros de fermentación y controlar los olores de forma más adecuada (Negro et al., 2000).

Este tema, toma vigencia en el Perú, dado que la falta de cultura ambiental y la ineficiente gestión de residuos han generado que la contaminación de ciertas ciudades sea uno de los problemas más importantes que afronta como nación. Según cifras aportadas por el Ministerio del Ambiente (2016), ya para el año 2014 se generaron un total de 7.497.482 toneladas de residuos urbanos municipales. En promedio, se produjeron 13.244 t/día de residuos sólidos, lo que representa una generación de 4.834.060 toneladas en el año.

Se puede evidenciar entonces, que en el Perú existe un problema ambiental a causa de la basura generada. Para disminuir estos problemas dentro del país, algunas municipalidades presentan diferentes programas, planes y caracterizaciones como parte de una gestión ambiental que ayudan a identificar, determinar la cantidad de residuos y sus posibles tratamientos. Tal es el caso de la municipalidad de Tambogrande, donde el $90 \%$ de residuos sólidos son recolectados y trasladados por un camión volquete en mal estado hacia el Botadero Municipal, ubicado en una zona inundable contaminando el ambiente.

Como resultado de ello, se evidencia la necesidad de instalar una planta de tratamiento para los residuos orgánicos, de esta manera se tendría una razón de peso para convencer a la población sobre la separación de los residuos en el hogar y aspirar a un desarrollo sustentable. A este respecto, se considera que una planta de compostaje es una instalación destinada al reciclaje de los residuos orgánicos mediante un tratamiento biológico de los mismos, dando como resultado un compost o abono orgánico (López, 2017).

En correspondencia con lo planteado, el objetivo de este artículo es la propuesta de la instalación de una planta de tratamiento de residuos sólidos en la 


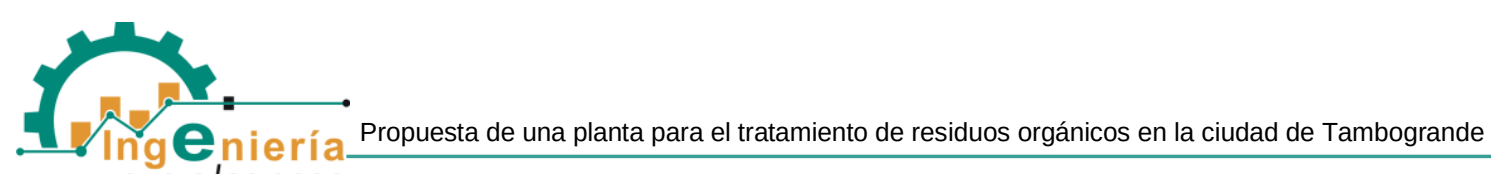

ciudad de Tambogrande, como una gestión de los residuos orgánicos, entendida como el manejo de todas aquellas actividades que tengan como propósito minimizar los impactos de los residuos sólidos en la salud, el ambiente y lo estético, la cual podría tener un impacto directo en la calidad de vida de las poblaciones.

\section{MÉTODO}

El tipo de investigación aplicada fue proyectiva, con un diseño no experimental, transeccional y de campo, puesto que se utilizó el conocimiento para la propuesta de instalación de la planta de tratamiento de residuos, la determinación de los equipos necesarios, la distribución de la planta en el sitio y la gestión para determinar la inversión requerida. Al respecto, Hurtado (2010) la define como aquella que intenta proponer soluciones determinadas a partir de un proceso previo de indagación que implica, explorar, describir y proponer alternativas de cambio, mas no necesariamente la ejecución de la propuesta.

El estudio se contextualizó en la ciudad de Tambogrande, que es un Distrito Rural integrado por 151 caseríos, 02 Centros Urbanos (capital Distrital y Cruceta) y 04 Comunidades Campesinas. En una parte de su territorio se encuentran establecidas 05 Municipalidades de Centro Poblado (La Peñita, Valle de los Incas, San Martín CP3, Tejedores y Cruceta).

\section{RESULTADOS}

Según el estudio integral para el mejoramiento del sistema de recolección y disposición de los desechos sólidos en la Ciudad de Tambogrande, se producen 0,487 kg. de basura por habitante diario. En la figura 1 se observa que, la composición de la basura es mayormente de residuos orgánicos, aproximadamente en un $80 \%$.

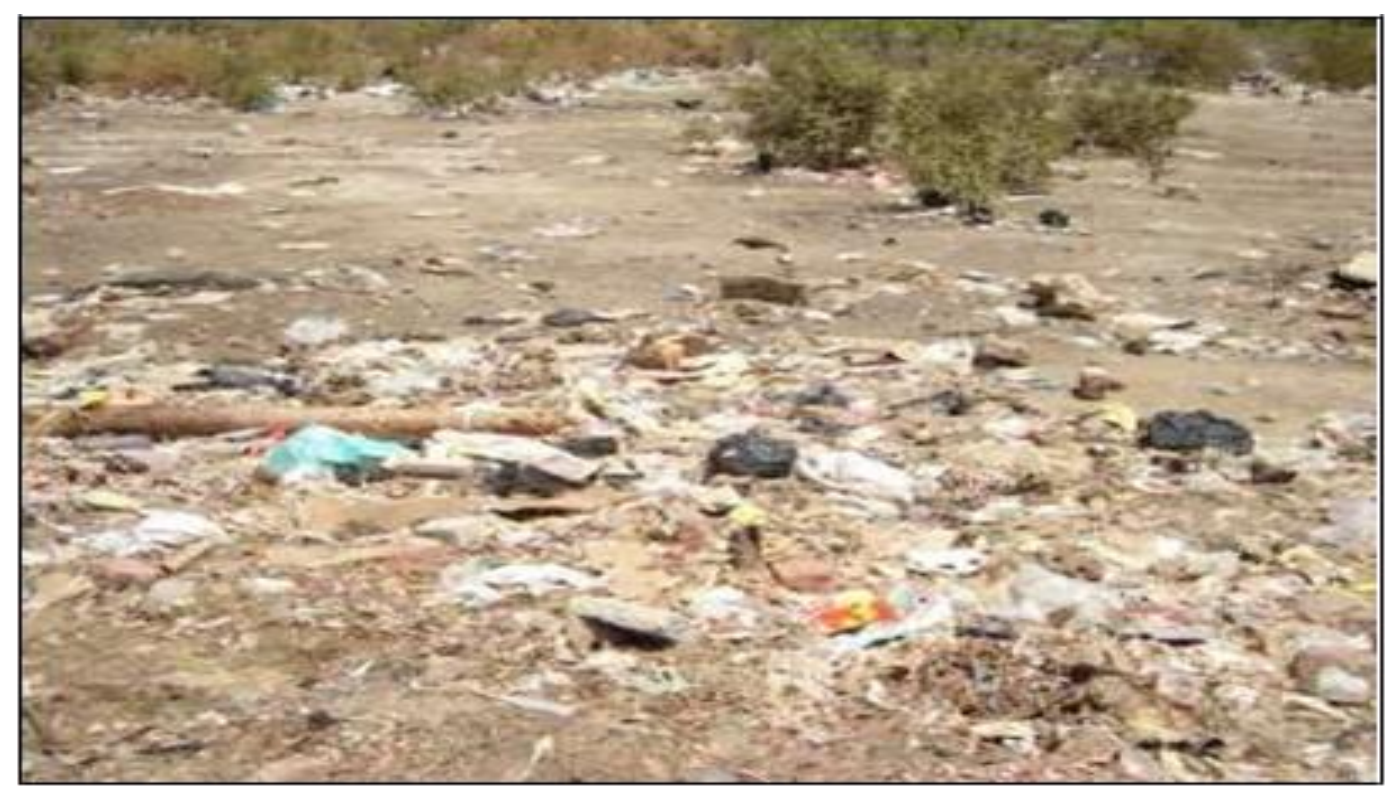

Figura 1. Basura depositada en zonas aledañas al mercado principal de la ciudad de Tambogrande 
Para hacer frente a esta situación, el compostaje se presenta como la alternativa más viable para la gestión de los residuos sólidos, con unos costos de inversión y de operación menor que en la incineración. Además, se consideró que el proceso de compostaje provoca menos impactos ambientales que la incineración y el depósito en vertederos. Así pues, el tratamiento de los residuos domiciliarios y agropecuarios mediante su compostaje, contribuye claramente a cumplir los objetivos establecidos en las normativas vigentes, ya que reduce la cantidad de residuos generados en la ciudad de Tambogrande.

De manera que, el compostaje es un proceso biológico aerobio, que bajo condiciones de aireación, humedad y temperaturas controladas y combinando fases mesófilas (temperatura y humedad medias) y termófilas (temperatura superior a $45^{\circ} \mathrm{C}$ ), transforma los residuos orgánicos degradables, en un producto estable e higienizado, aplicable como abono o sustrato (Negro et al., 2000).

Ahora bien, a objeto de esta investigación el diagnóstico realizado evidenció que actualmente y de modo particular en la ciudad de Tambogrande, la información estadística que se tiene no es suficiente, además de estar desactualizada para estimar los volúmenes de venta y de consumo efectivo de compostaje. Se conoció además que, dentro de los fertilizantes sintéticos, el producto más usado es la urea, siguiéndole en orden de importancia, el sulfato de amonio y el nitrato de amonio.

Las fuentes principales de fertilización orgánica están constituidas por el guano de la isla y el estiércol. El primero usado en el Valle del Chira; Alto Piura y San Lorenzo, aunque también se usa el compost, pero en pequeñas cantidades y solo para uso particular, en cambio, el Valle del Medio y Bajo Piura han restringido su fertilización exclusivamente al uso de la urea. En cuanto al uso de fertilizantes orgánicos, es importante el conocimiento que se tiene de los mismos, lo que falta al parecer es insistir y capacitar a los potenciales usuarios sobre las bondades de sus propiedades para incrementar su uso.

Respecto a la oferta de abonos orgánicos, la búsqueda de información en toda la región es de $7000 \mathrm{TM}$ al año correspondiente al humus de lombriz. En cuanto a los fertilizantes, el principal nutrimento que se demanda es el nitrógeno, el mismo que se encuentra en la urea, sulfato de amonio y nitrato de amonio en grandes cantidades.

De igual manera, se pudo apreciar que el mercado del compostaje procedente de residuos municipales y agropecuarios tiene un gran futuro, centrándose tanto en el sector agrícola como en sus actividades asociadas (ganadería). Es así que las tendencias del mercado actual son hacia una agricultura orgánica.

Estos productos representan una alternativa rentable para las economías regionales, ya que permiten apoyarse en las ventajas comparativas que presenta cada zona, compensando así los mayores costos de insumos y fletes, y constituyéndose, por lo tanto, en una de las posibilidades que se perfilan como más promisorias para la provincia de Piura.

\section{Propuesta}

Para la planta de tratamiento de residuos orgánicos se cotizaron los equipos idóneos para realizar el proceso de 
L Ing eniería. Propuesta de una planta para el tratamiento de residuos orgánicos en la ciudad de Tambogrande

compostaje (Cuadro 1), el cual se inicia con la entrada a la planta del camión recolector de basura y camiones de las agroexportadoras de mangos. Cabe resaltar que se llama "Mango de Protocolo de corte" a aquel mango que una vez llegada a la planta de empaque, pasa por una evaluación de la autoridad sanitaria local (SENASA), quien realiza un protocolo de corte para evaluar la calidad fitosanitaria de la fruta $y$ hace que ese mango sea desechado (Figura 2).

Cuadro 1. Equipamiento de centro de producción de compostaje

\begin{tabular}{lccrr}
\hline Rubro & Unidad & Cant. & Precio & Costo Total \\
\hline 1. Maquinarias y Equipos & & & & $\mathbf{\$ 4 9 , 9 4 1 . 0 0}$ \\
\hline 1.1.Minicargador Bobcat multiuso & Equipo & 1.0 & $\$ 30,000.00$ & $\$ 30,000.00$ \\
1.2.Picadora & Equipo & 1.0 & $\$ 7,482.89$ & $\$ 7,482.89$ \\
1.3.Zaranda & Equipo & 1.0 & $\$ 576.00$ & $\$ 576.00$ \\
1.4.Línea de segregación & Equipo & 1.0 & $\$ 10,808.11$ & $\$ 10,808.11$ \\
1.5.Máquina de coser sacos & Equipo & 1.0 & $\$ 159.00$ & $\$ 159.00$ \\
1.6.Herramientas básicas $\left({ }^{*}\right)$ & Global & 1.0 & $\$ 915.00$ & $\$ 915.00$ \\
\hline & & & TOTAL & $\mathbf{\$ 4 9 , 9 4 1 . 0 0}$ \\
\hline
\end{tabular}

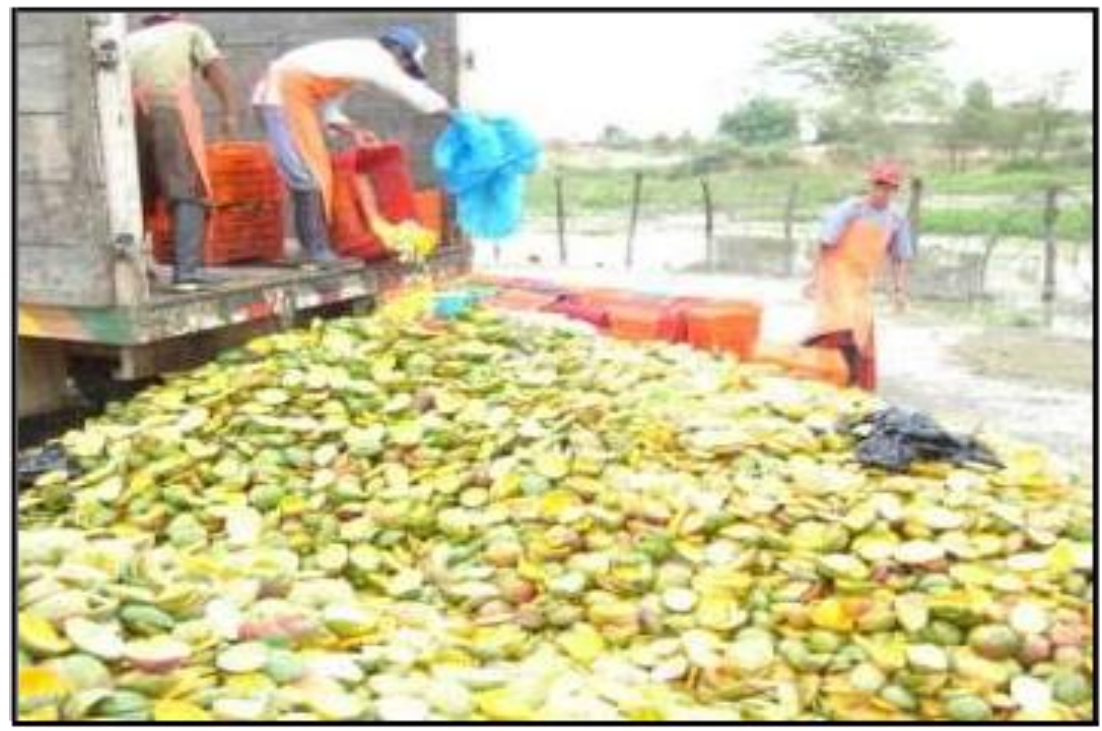

Figura 2. Personal descargando mango de descarte en la planta norte compostaje.

El objetivo primordial que persigue la distribución en planta es hallar un ordenamiento de las áreas de trabajo y del equipo, que sea la económica para el trabajo, al mismo tiempo segura y satisfactoria para los empleados. Se utilizó una distribución por proceso o por fusión, dado que, todas las operaciones del mismo proceso estaban agrupadas, la maquinaria es pesada y también existe un solo producto, lo que permite un mejor manejo de material y facilidad del control del proceso. 


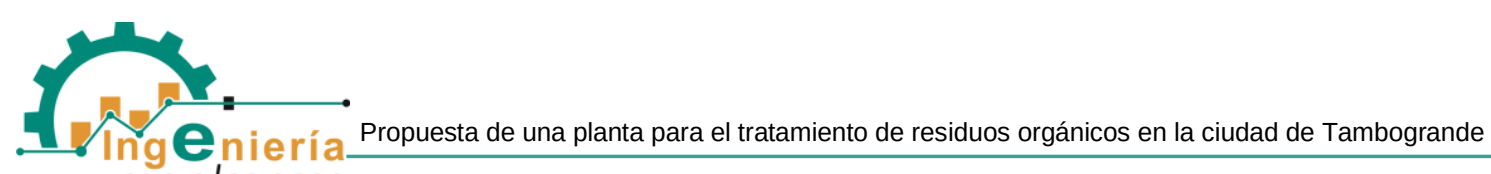

También se contempló la cantidad de suministro, para ello se pesa en el caso de las agroexportadoras de mango, una "jaba de mango" con una balanza de mano y luego se multiplica por el número de jabas recepcionadas. Posteriormente, los camiones se retiran y un trabajador se encarga de llevar los residuos del camión recolector a la faja transportadora, en donde dos operarios se encuentran a ambos lados de la faja transportadora, clasificando los residuos inorgánicos (Figura 3).
Una vez realizada la selección de estos materiales, se llevan a la picadora, los cuales son triturados y almacenados en mantas; para que luego el minicargador frontal se encargue de llevar los materiales hasta el área de compostaje en donde se procede a la mezcla homogénea de los materiales, tales como bazofia, flores secas del cementerio, mango de descarte, hojas y ramas recolectados de las podas de los parques de la ciudad, papel y agua.

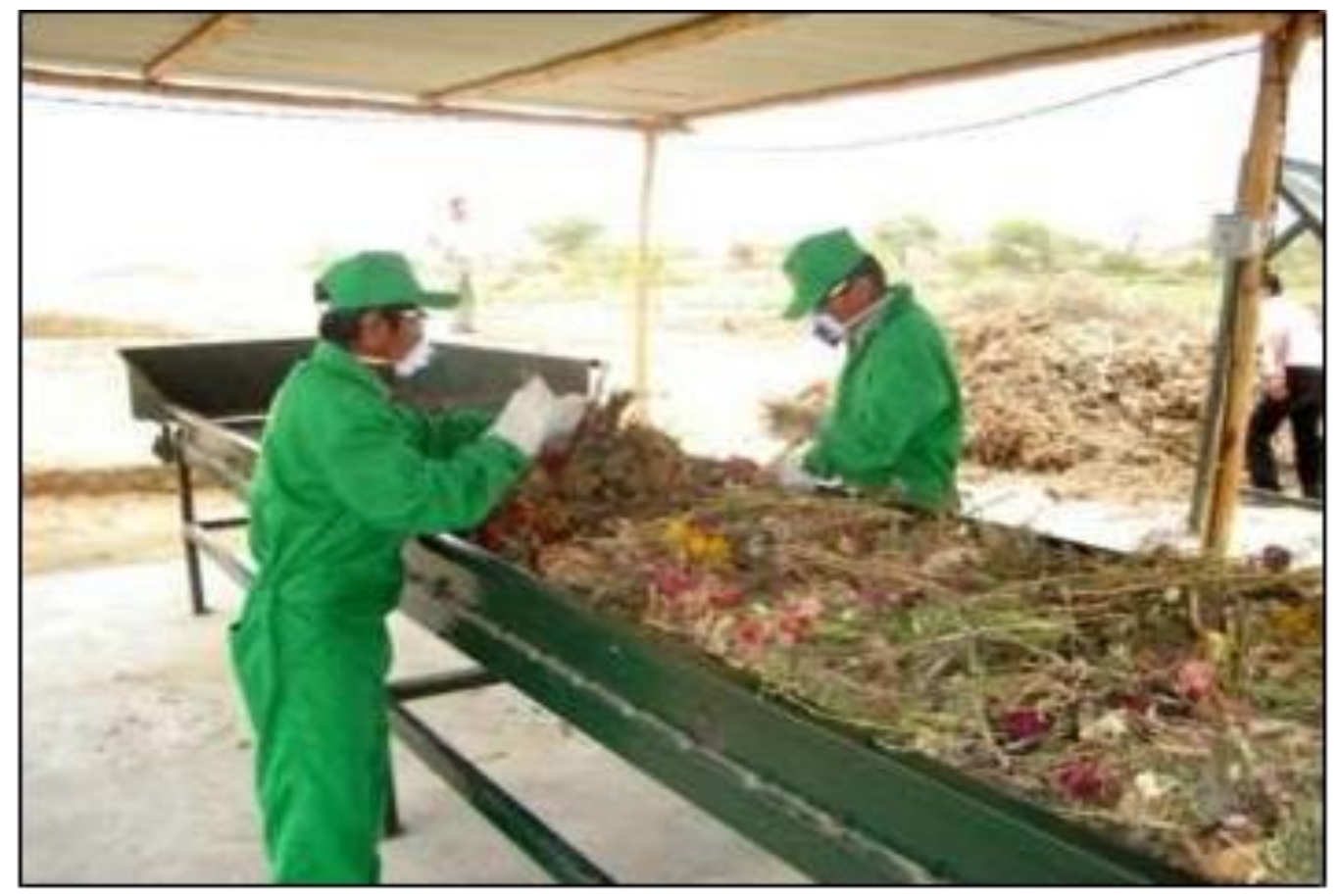

Figura 3. Personal de la planta norte compost clasificando los residuos.

Luego se ejecutó el armado de la pila piramidal con altura 1,30 $\mathrm{m}$ y base de 2,04 $\mathrm{m}$, para lo cual, utilizando una brújula se orientó con el norte magnético, de esa manera se asegura un óptimo aprovechamiento de la luz solar por la pirámide. En seguida, se trazó el cuadrado base, colocándose una estaca en cada uno de los vértices (Figura 4). 
Propuesta de una planta para el tratamiento de residuos orgánicos en la ciudad de Tambogrande

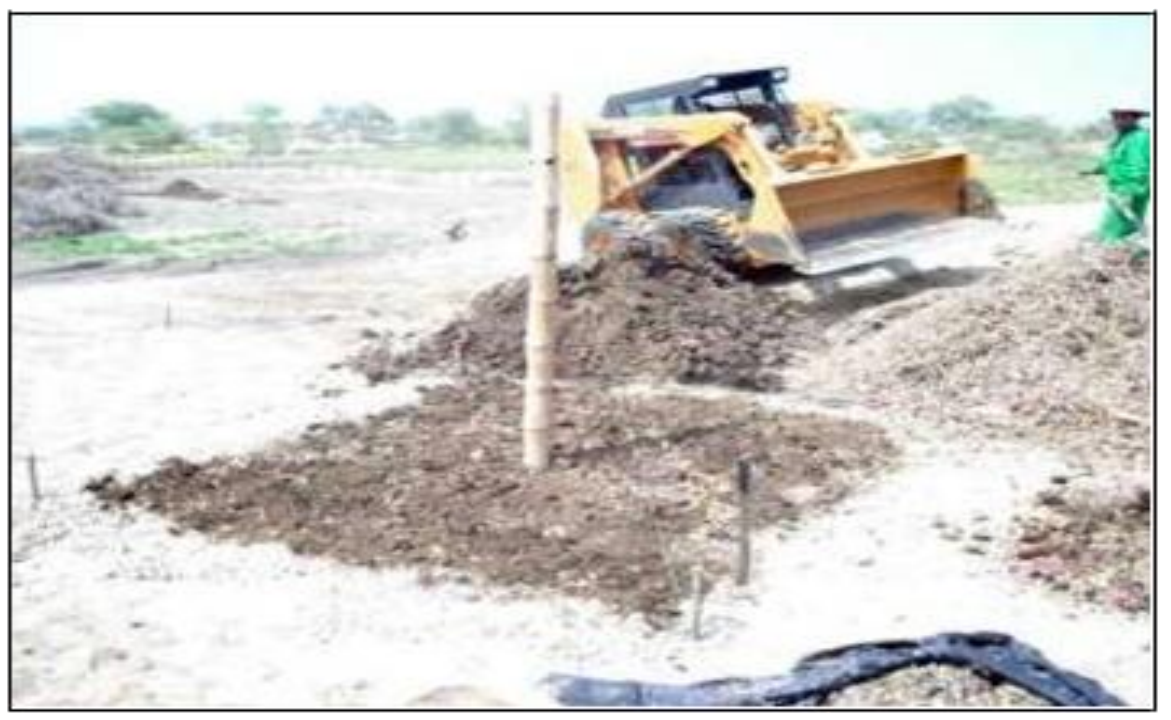

Figura 4. Armado de base de pila piramidal en la planta norte de compostaje.

En el centro del cuadrado se colocó una caña de Guayaquil de 1,50 m de altura. Esta caña se ajustó con cordeles de nylon amarrados a las estacas, de manera que conformaran el armazón de la pirámide. Una vez realizado esto, se ubicó el material en la pirámide secuencialmente en forma de capas, guiándose por la estructura previamente armada. Al terminar de construir la pirámide, se retiró la caña con mucho cuidado para que no sufra daños en su infraestructura. El espacio vacío sirve como chimenea para los gases producidos en la reacción de los materiales. Las proporciones que se utilizan en una pirámide de compostaje son:

- 676 kilogramos de bazofia o estiércol que equivalen al 49,2 \% de la pirámide.
- 624 kilogramos de mango que equivalen al $45,4 \%$ de la pirámide.

- 75 kilogramos de flores secas que equivalen al 5,5 \% de la pirámide.

- Además, se utilizan 114 litros de agua para dicho proceso.

Toda esta pirámide tiene un peso de aproximadamente 1489 kilogramos. Luego se dejó durante 30 días o menos en algunos casos, ya que el intenso sol de Tambogrande ayuda rápidamente a la degradación de estos residuos orgánicos. Para ver su evolución, se va midiendo temperatura en el transcurso de los días (Figura 5). 

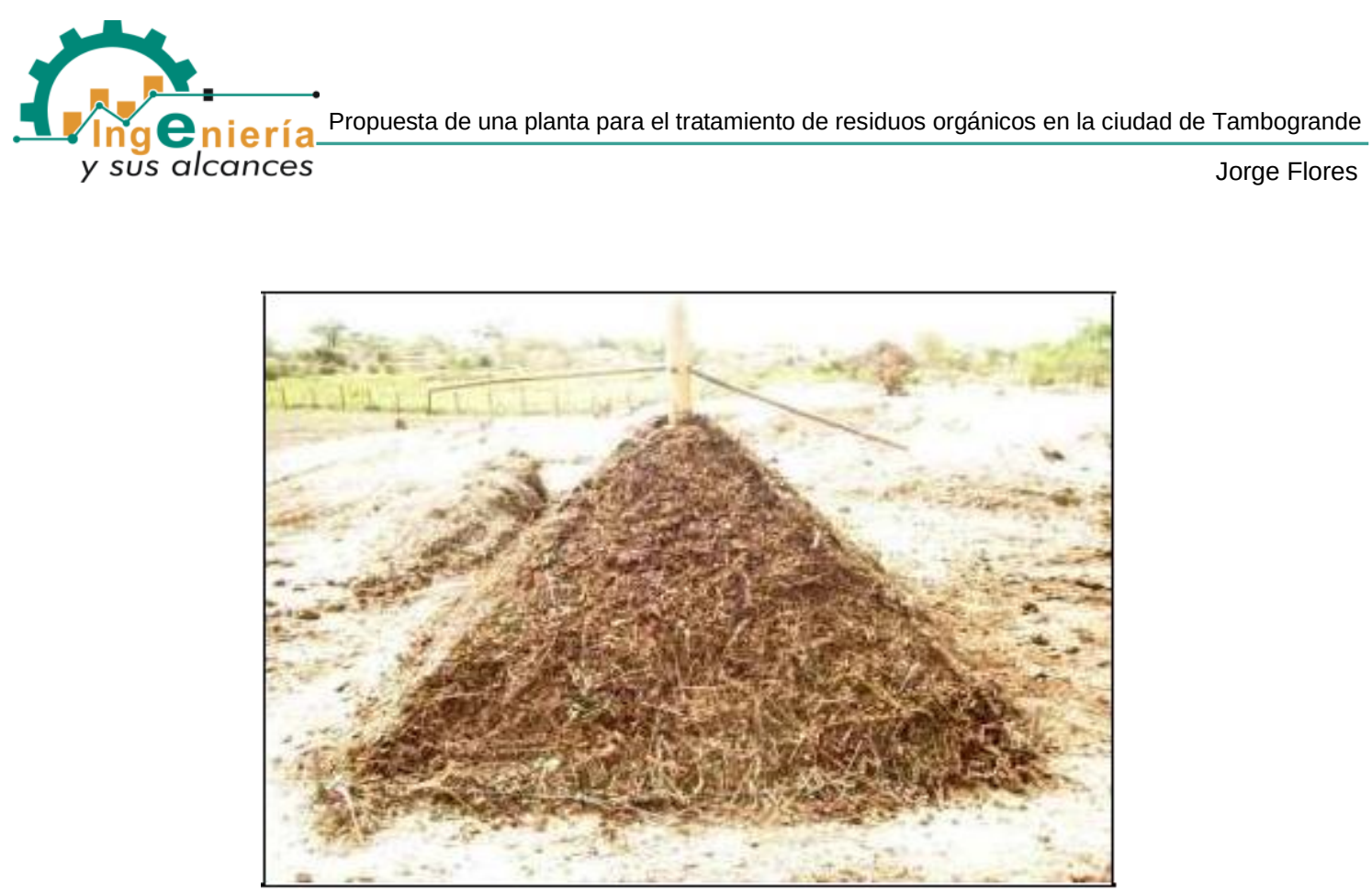

Figura 5. Pila piramidal en la planta norte de compostaje.

Después de transcurridos los días, una semana antes de llevarla a la sección de cribado, la pila piramidal se orea antes. Una vez en el área de cribado, con la zaranda se realiza la separación de los residuos a fin de quitar los desechos inorgánicos aún presentes y el material que no ha terminado el proceso de compostaje. Este material no degradado es devuelto para formar nuevas pirámides sirviendo de inóculo para la fermentación (Figura 6).

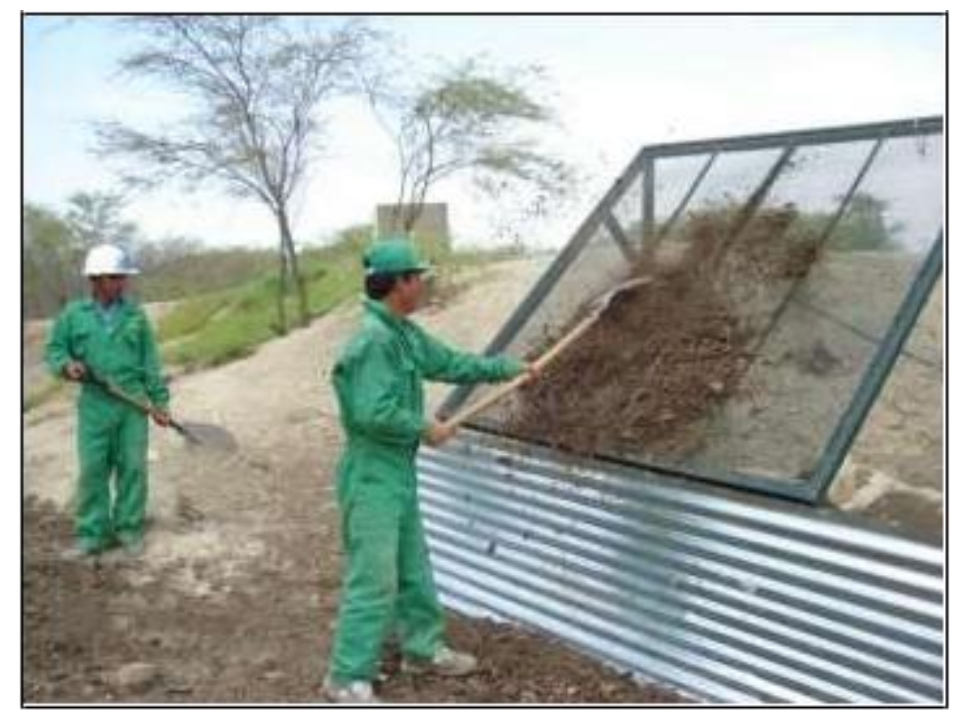

Figura 6. Cribado en la planta norte de compostaje.

Una vez cumplida la fase de cribado, el material es llevado en carretillas para su posterior empaque (Figura 7). Cabe resaltar que el control de calidad de este producto se realiza cuando se ha procesado un lote de 20 a 30 toneladas de compost. Todo el proceso de armado de una pirámide dura aproximadamente 2 horas y 20 minutos y en toda la jornada se realizan unas 04 pilas piramidales diarias. 
Ahora bien, con el apoyo del laboratorio de la Universidad Nacional de Piura de la Facultad de Ingeniería Química, se realizaron las determinaciones analíticas. Para ello, se llevó a cabo una comparación de resultados con los valores de referencia europeos recogidos en la legislación existente; en cuanto a algunos de los parámetros para los que no existen valores de referencia en dicha legislación, se han tomado estándares de otros países con el fin de poder contrastar los resultados que se obtuvieron.

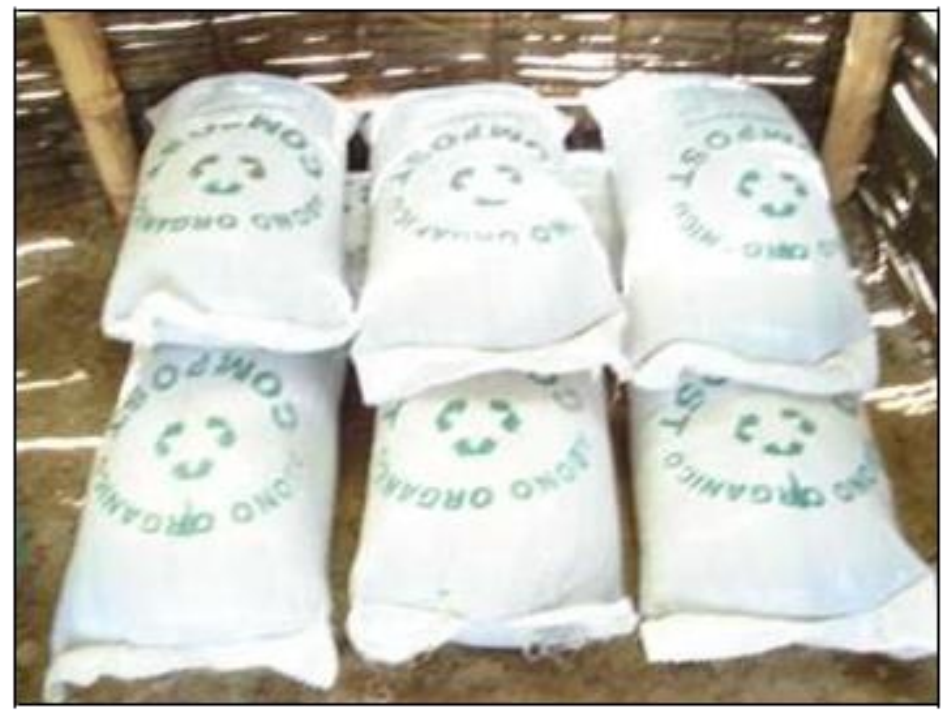

Figura 7. Empacado en la planta norte de compostaje.

En tal sentido, fue evidente que la calidad del compostaje viene definida en primer lugar por las características del material utilizado. Esto implica la realización de recogida selectiva de la fracción orgánica de los residuos sólidos urbanos $\mathrm{y}$ agropecuarios. Por tanto, el proceso de compostaje debe realizarse de forma adecuada para obtener un producto final que cumpla los requisitos exigidos por la legislación vigente, evitando la contaminación por metales pesados y otro tipo de compuestos, una vez el producto sea utilizado (Röben, 2002).

Para la instalación de la planta se requiere una inversión de $\$ 1,500.00$ dólares en el primer año, los cuales servirán para la compra de 02 extintores, 01 termómetro digital, 01 medidor de $\mathrm{pH}$ y 01 balanza digital. Mientras que, en los años siguientes se considerará una inversión de \$1,000.00 dólares, los cuales se utilizarán para la renovación anual de uniformes, botas, herramientas y equipos de protección personal, así como de mantenimiento de la infraestructura.

La planta trabajará 300 días al año, considerando 36 días para reparaciones y limpieza general. En esta planta se producirán 1.200 toneladas de compostaje al año, y dado que, cada mes se procesarán 100 toneladas, se trabajará en turnos de 8 horas al día para que esta producción se logre, variando en el período que abarca desde diciembre hasta abril. Debido a la campaña de mango donde se estima que la producción 


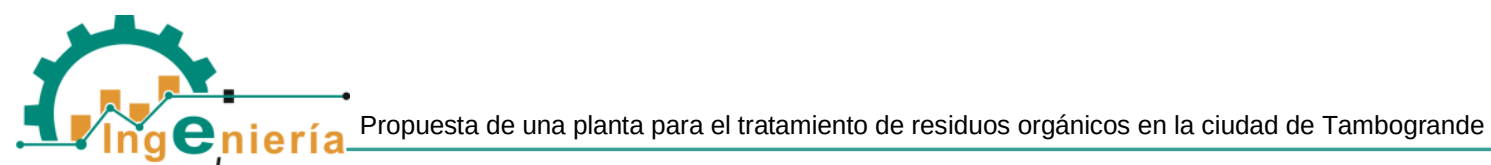

aumentará considerablemente, teniendo estimaciones de 240 toneladas al mes.

En relación con las implicaciones ambientales, el compostaje tiene efectos positivos y relativamente duraderos para mejorar el suelo; no obstante, también puede tener fácilmente un impacto perjudicial sobre el medio ambiente. Las materias de partida del compostaje, concretamente basura doméstica, pueden contener sustancias que en altas concentraciones generarían un efecto nocivo para el suelo y las plantas, y en último término para el consumidor a través de la cadena alimenticia (animales, seres humanos). Especial importancia tienen en este contexto los metales pesados.

Una vez implementada la propuesta, es necesario llevar a cabo el programa de capacitación del personal y de la comunidad. Para ello, las capacitaciones se desarrollan en dos niveles, los cuales se describen a continuación:

El primero se refiere al desarrollo de capacidades organizacionales para los trabajadores de las PYME de producción de compost, donde se abordarán los temas de desarrollo personal y liderazgo.

El segundo nivel está dirigido a los productores e instituciones de la comunidad donde se ejecutarán las actividades de producción y utilización del compostaje.

La metodología utilizada comprendió talleres informativos y de campo, los cuales fueron realizados en la misma planta. Para el desarrollo de estos talleres, se contó con un campo demostrativo de $500 \mathrm{~m}^{2}$. Además, se realizaron capacitaciones para preparar la implementación de un sistema de preselección de la materia orgánica en el mercado municipal y algunas empresas agrícolas (Figuras 8 y 9).

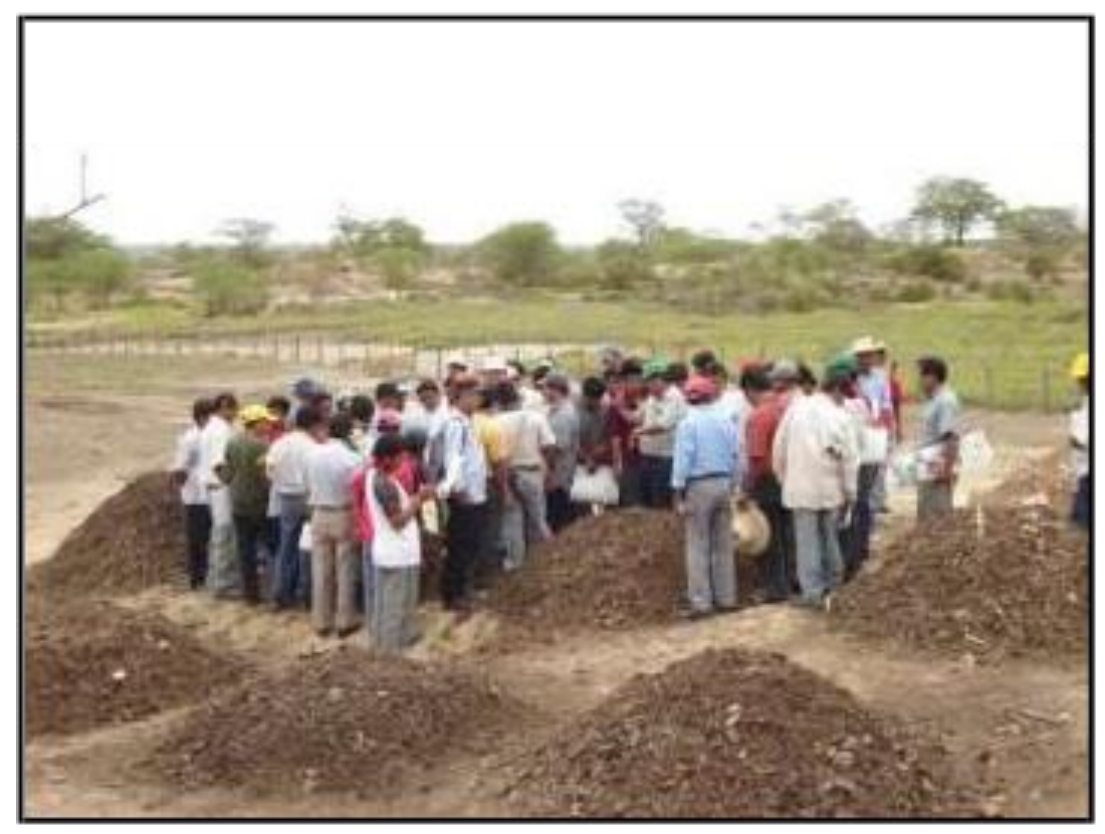

Figura 8. Talleres de campo a agricultores. 


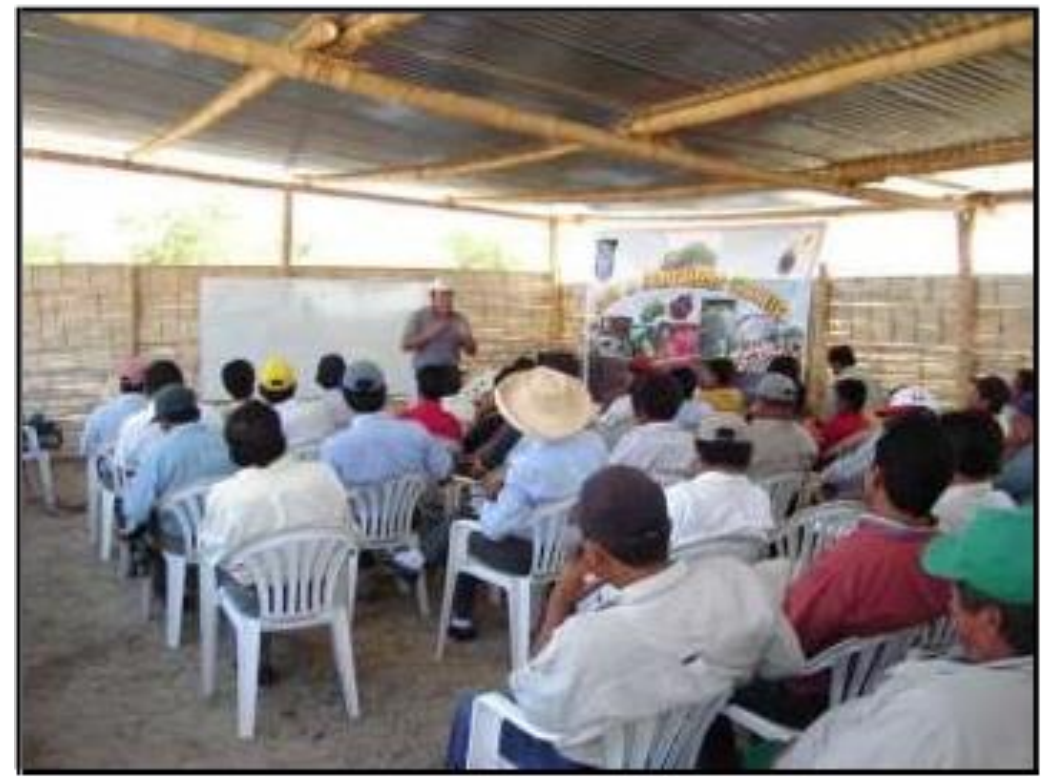

Figura 9. Talleres en el centro de capacitación a agricultores.

El proyecto buscó la equidad de género permitiendo el acceso al conocimiento sobre el proceso de compostaje y sus ventajas, ya que tiene el alto potencial de producir un cambio de conducta contribuyendo al desarrollo local. Asimismo, los niños, profesoras y profesores de los centros educativos pueden usar el proyecto como modelo de enseñanza.

Finalmente, es importante acotar que la empresa encargada de llevar a cabo la presente propuesta, estará conformada por una directiva que aglutinará representantes de diferentes instituciones de la sociedad civil y una ejecutiva con un personal mínimo recomendado de 6 trabajadores para garantizar la operatividad de la planta de compostaje.

\section{CONCLUSIONES}

La instalación de una planta de tratamiento para los residuos sólidos municipales es deseable, de esta manera se tendría una razón de peso para convencer a la población sobre la separación de los residuos en el hogar y aspirar a un desarrollo sustentable. Esta fue la idea principal del presente artículo: visualizar la factibilidad de la instalación de una planta de tratamiento de los residuos sólidos municipales, analizando su proceso, del cual se concluye:

La utilización del método piramidal para la producción de compost se consideró como el más apropiado por razones de tiempo, rendimiento y economía.

El proceso de fabricación del compost debe controlarse como cualquier otro sistema de producción; si se trata de materiales con características no adecuadas se generarán problemas durante el proceso $\mathrm{y}$ en la calidad del producto final. $\mathrm{Si}$ no se controla el proceso, se afectará la calidad final, pero también aumentará la probabilidad de tener problemas de generación de malos olores y lixiviados.

Del análisis de la muestra de compost de la planta, se evidenció un nivel aceptable de acuerdo a los parámetros de Kiehl. 


\section{REFERENCIAS}

Barradas, A. (2009). Gestión integral de residuos sólidos municipales: estado del arte. Gytsu, Miantilán, Veracruz, México

Hurtado, J. (2010). Metodología de la Investigación Holística. SYPAL-IUTC. Venezuela

López, M. (2017). Manual. Tratamiento de residuos urbanos o municipales (UF0285). Certificados de profesionalidad. Gestión de residuos urbanos e industriales. EDITORIAL CEP. Madrid
Ministerio del Ambiente (2016). Plan Nacional de Gestión Integral de Residuos Sólidos, MINAM, Perú, Inf.Téc, Resolución Ministerial № 191-2016MINAM

Negro, M. J., Villa, F., Aibar, J., Aracón, R., Ciria, P., Cristóbal, M. V. y Lacasta, C. (2000). Producción y gestión del compost. Disponible en: http://digital.csic.es/handle/10261/1 6792

Röben, E. (2002). Manual de compostaje para municipios. Loja, Ecuador: DED, Ilustre Municipalidad de Loja, 68 